\title{
CONSTRUCTIONS PRESERVING THE ASSOCIATIVE AND THE COMMUTATIVE LAWS
}

\author{
A. SHAFAAT
}

(Received 27 September 1974; revised 8 January 1975)

\begin{abstract}
The associative and the commutative laws are characterized by preservation under the construction of powers and addition of a (new) unit element. This is used to generate the varieties defined by the two laws from two element groupoids.
\end{abstract}

Let $\mathscr{G}$ be the category of all groupoids (that is, algebras with one binary operation) and groupoid homomorphisms. By a functional construction on groupoids we mean a functor $F$ from the "power category" $\mathscr{G}^{I}$ into $\mathscr{G}$. We say that a class $\mathscr{K}$ of groupoids is $F$-closed or closed under $F$ if for every family $\left(A_{i}\right)_{i \in l}$ of groupoids of $\mathscr{K}$ the groupoid $F\left(\left(A_{i}\right)_{i \in I}\right)$ belongs to $K$. A set $\Sigma$ of groupoid identities will be said to be preserved under $F$ if the variety defined by $\Sigma$ is $F$-closed.

In this note we shall be concerned with two functional constructions $-P$ and ()$^{0}-$ defined as follows. For every groupoid $(A,+)$ we define $P((A,+))$ to be the groupoid $(P(A),+)$, where $P(A)$ is the set of all subsets of $A$ and $X+Y=\{x+y ; x \in X, y \in Y\}$ for all $X, Y \subseteq A$. If $f:(A,+) \rightarrow(B,+)$ is a groupoid homomorphism then we define $P(f)$ to be the function from $P(A)$ to $P(B)$ which sends $X \subseteq A$ to its image under $f$. It is easily seen that $P(f)$ is a homomorphism from $(P(A),+)$ to $(P(B),+)$ and that the function $P: \mathscr{G} \rightarrow \mathscr{G}$ so defined is a functor. We shall refer to $P$ as the power functor. To define ()$^{\circ}: \mathscr{G} \rightarrow \mathscr{G}$ let $(A,+)$ be an arbitrary groupoid. We define $((A,+))^{0}$ to be the groupoid $\left((A)^{0},+\right)$ obtained from $(A,+)$ by adding a new unit element 0 ; that is, $(A)^{0}$ consists of the disjoint union of $A$ and $\{0\}$ and $a+0=0+a=a$ for all $a \in(A)^{0}$. For a groupoid homomorphism $(A,+) \stackrel{f}{\rightarrow}(B,+)$ we define $(f)^{0}$ to be the function from $(A)^{0}$ into $(B)^{0}$ such that $(f)^{0}$ restricted to $A$ is $f$ and 
$(f)^{0}(0)=0$. Again it is easy to see that $(f)^{0}$ is a homomorphism and ()$^{0}$ is a functor. We shall refer to ()$^{\circ}$ as the addition-of-unit functor.

The associative and the commutative laws (i.e., the identities $x+(y+z)=$ $(x+y)+z$ and $x+y=y+x)$ are preserved under $P$ and ()$^{\circ}$, as can be verified without difficulty. Our first theorem shows that apart from the expected case of the identity $x=x$ (which is preserved under all constructions on groupoids) $x+y=y+x$ and $x+(y+x)=(x+y)+z$ are the only identities preserved under our two constructions.

THEOREM 1. Let $\mathscr{V}$ be a variety of groupoids. Then $\mathscr{V}$ is closed under $P$ and ()$^{\circ}$ if and only if $\mathscr{V}$ is one of the following four varieties:

(1) the class of all groupoids

(2) the class of all commutative groupoids

(3) the class of all semigroups

(4) the class of all commutative semigroups.

It is clear from the above theorem that the class of all commutative semigroups may be characterized as the smallest variety of groupoids closed under the power and the addition-of-unit functors. The following theorem gives a sharper result.

THEOREM 2. The variety of commutative semigroups is the smallest variety of groupoids closed under the power functor $P$.

Before turning to the proofs of the above two theorems we make two remarks and give some necessary definitions.

Remark 1. A variety $\mathscr{V}$ of groupoids is closed under $P$ and ()$^{\circ}$ if and only if $\mathscr{V}$ is closed under either of the compositions ()$^{0} P: \mathscr{G} \stackrel{P}{\rightarrow} \mathscr{G} \stackrel{()^{0}}{\longrightarrow} \mathscr{G}$ and $P()^{0}: \mathscr{G} \stackrel{\mathfrak{1}^{\circ}}{\longrightarrow} \mathscr{G} \stackrel{P}{\rightarrow} \mathscr{G}$. If we write $P^{0}=P()^{0}$ then Theorem 1 determines all the $P^{0}$-closed varietes of groupoids.

REMARK 2. The set of all varieties closed under a functional construction $F$ is a complete sublattice $L_{F}$ of the complete lattice $L$ of all varieties of groupoids. Theorem 1 describes $L_{P^{0}}$ as the four-element Boolean lattice while Theorem 2 determines the "zero element" of the complete lattice $L_{P}$.

In the sequel we shall of ten denote a groupoid $(A,+)$ simply by $A$.

Let $X$ be a fixed infinite set. Let $W_{X}$ denote the word groupoid (cf. Cohn (1965)) on $X$. We say that a word $w \in W_{x}$ is linear if every "variable" $x \in X$ occurs at most once in $w$. An identity $v=w$ will be called linear if $v$ and $w$ are both linear. (For example, $(x+y)+z=x+(y+z)$ and $x+y=y+x$ are 
linear). An identity is called regular if every variable that occurs on one side of it also occurs on the other.

To prove Theorem 2 we need the following result which follows from Whitney (1974) or Bleicher, Schneider and Wilson (1973).

Theorem 3. A variety is P-closed if and only if is definable by a set of regular, linear identities.

PROOF OF THeOREM 2. If $v\left(x_{1}, \cdots, x_{n}\right)=w\left(x_{1}, \cdots, x_{n}\right)$ is a regular linear identity then under the associative and the commutative laws it reduces to $\left(x_{1}+\cdots+x_{n}\right)=\left(x_{1}+\cdots+x_{n}\right)$. Hence every commutative semigroup satisfies every regular, linear identity. In view of Theorem 3 , this proves Theorem 2 .

We now give some lemmas directed towards the proof of Theorem 1.

Let $v\left(x_{1}, \cdots, x_{n}\right) \in W_{X}$. Then $v\left(x_{1}, \cdots, x_{n-1}, 0\right)$ is an element of $\left(W_{X}\right)^{0}$. It is immediate that if $n>1$ and $v$ properly involves $x_{1}, \cdots, x_{n}$ then $v\left(x_{1}, \cdots, x_{n-1}, 0\right) \in W_{x}$. Also if $v\left(x_{1}, \cdots, x_{n}\right)$ is linear then so is $v\left(x_{1}, \cdots, x_{n-1}, 0\right)$.

The following lemma hardly needs any proof.

Lemma 1. A necessary and sufficient condition for a variety $\mathscr{V}$ to be ()$^{0}$-closed is that if $v\left(x_{1}, \cdots, x_{n}\right)=w\left(x_{1}, \cdots, x_{n}\right)$ is an identity of $\mathcal{V}$ and $v\left(x_{1}, \cdots, x_{n-1}, 0\right) \in W_{X}$ then $w\left(x_{1}, \cdots, x_{n-1}, 0\right) \in W_{X}$ and $v\left(x_{1}, \cdots, x_{n-1}, 0\right)=$ $w\left(x_{1}, \cdots, x_{n-1}, 0\right)$ is an identity of $\mathscr{V}$.

COROllary 1. Every identity of a ( $)^{0}$-closed variety is regular.

Lemma 2. Let $v\left(x_{1}, \cdots, x_{n}\right)=w\left(x_{1}, \cdots, x_{n}\right)$ be a regular linear identity such that the variables $x_{1}, \cdots, x_{n}$ occur in the order $x_{1}, \cdots, x_{n}$ both in $v$ and $w$. Then:

The associative law $(x+y)+z=x+(y+z)$ implies $v=w$.

If $v=w$ is an identity of $a()^{0}$-closed variety $\mathscr{V}$ and $v, w$ are distinct then $(x+y)+z=x+(y+z)$ is also an identity of $\mathscr{V}$.

Proof. The first part (2.1) of the lemma is fairly clear. For the second part we use induction on the number $n$ of variables occurring in $v=w$. If $n=1$ then regularity and linearity of $v=w$ implies that $v, w$ are identical variables. Hence the required result (2.2) is true vacuously. Let $n>1$ so that we can write $v=v_{1}+v_{2}, w=w_{1}+w_{2}$ for some (linear) words $v_{1}, v_{2}, w_{1}, w_{2} \in W_{x}$. Let $v, w$ be distinct and let $v=w$ be an identity of $\mathscr{V}$. If $v_{1}, w_{1}$ involve the same variables then so do $v_{2}, w_{2}$ and by setting all the variables in $v_{2}$ equal to 0 and using Lemma 1 we see that $v_{1}=w_{1}$ is a regular linear identity of $\mathscr{V}$. Similarly, $v_{2}=w_{2}$ is also an identity of $\mathscr{V}$ and since $v_{1}+v_{2}, w_{1}+w_{2}$ are distinct therefore either $v_{1}, w_{1}$, or $v_{2}, w_{2}$ are distinct. We can thus use the induction hypothesis to conclude that $(x+y)+z=x+(y+z)$ is an identity of $\mathscr{V}$. 
Let now one of $v_{1}, w_{1}$ (say, $v_{1}$ ) involve a variable (say, $x_{i}$ ) which does not occur in $w_{1}$. Then $n>i>1$ and if we substitute 0 for all the variables of $v=w$ except $x_{1}, x_{i}, x_{n}$ then we must get $\left(x_{1}+x_{i}\right)+x_{n}=x_{1}+\left(x_{i}+x_{n}\right)$. This proves the lemma.

We write a regular linear identity - as indeed we can-in the form $v\left(x_{1}, \cdots, x_{n}\right)=w\left(x_{f(1)}, \cdots, x_{f(n)}\right)$, where $f$ is a permutation on $\{1, \cdots, n\}$ and the variables $x_{1}, \cdots, x_{n}$ occur in $v$ in the order $x_{1}, \cdots, x_{n}$ and in $w$ in the order $x_{f(1)}, \cdots, x_{f(n)}$.

Lemma 3. Let $v\left(x_{1}, \cdots, x_{n}\right)=w\left(x_{f(1)}, \cdots, x_{f(n)}\right)$ be a regular, linear identity of a ()$^{0}$-closed variety $\mathscr{V}$. Then:

$$
\text { If } f(\mathrm{i}) \neq i \text { for some } i, 1 \leqq i \leqq n \text {, then } x+y=y+x \text { is an identity of } \mathscr{V} \text {. }
$$

Either $v=w$ is deducible from $x+y=y+x$ or $(x+y)+z=$ $x+(y+z)$ is an identity of $\mathscr{V}$.

Proof. Let $i$ be the smallest integer such that $1 \leqq i \leqq n$ and $f(i) \neq i$. Then $f(i)>i$. Substituting 0 for all the variables in $v=w$ except $x_{i}, x_{f(i)}$ we must have $x_{i}+x_{f(i)}=x_{f(i)}+x_{i}$, which gives (3.1), by Lemma 1 .

To prove (3.2) we use induction on $n$. If $n=1$ then $v=w$ is identical with $x=x$ and hence deducible from $x+y=y+x$. Let $n>1$ and assume (3.2) for all identities with less than $n$ variables. Clearly, we can write $v=v_{1}+v_{2}$, $w=w_{1}+w_{2}$. If $v_{1}, w_{1}$ involve the same variables then, as in the proof of Lemma 2 (2.2), we obtain the regular, linear identities $v_{1}=w_{1}$ and $v_{2}=w_{2}$ of $\mathscr{V}$. If the associative law does not hold for the groupoids of $\mathscr{V}$ then the induction hypothesis implies that $v_{1}=w_{1}, v_{2}=w_{2}$ are deducible from $x+y=y+x$, which shows that $v=w$ is also deducible from $x+y=y+x$.

We thus need to consider the case when one of $v_{1}, w_{1}\left(\mathrm{say}, v_{1}\right)$ involves a variable (say, $x_{i}$ ) not occurring in the other. If $f(1) \neq 1$ then, by (3.1), we can use commutativity to bring $x_{1}$ to the left most position in $w$. Hence we can assume $f(1)=1$ and therefore $1<i<n$. Substituting 0 for all of the variables $x_{1}, \cdots, x_{n}$ in $v=w$ except $x_{1}, x_{i}, x_{n}$ we must have $\left(x_{1}+x_{i}\right)+x_{n}=x_{1}+\left(x_{i}+x_{n}\right)$ or $\left(x_{1}+x_{i}\right)+x_{n}=x_{1}+\left(x_{n}+x_{i}\right)$ as identities of $\mathscr{V}$. In the case of $\left(x_{1}+x_{i}\right)+x_{n}=$ $x_{1}+\left(x_{n}+x_{i}\right)$ we have commutativity, by (3.1) (or by setting $x_{1}=0$ ). But commutativity and $\left(x_{1}+x_{i}\right)+x_{n}=x_{1}+\left(x_{n}+x_{i}\right)$ imply associativity. This proves (3.2) and the lemma.

Proof OF THEOREM 1. If $\mathscr{V}$ is one of the four varieties (1)-(4) mentioned in the theorem then, as already noted, $\mathscr{V}$ is closed under $P$ and ()$^{\circ}$.

Assume that $\mathscr{V}$ is closed under $P$ and ()$^{\circ}$. Then, by Theorem 3, $\mathscr{V}$ is defined $n !$ the set $\Sigma$ of all regular, linear identities of $\mathscr{V}$. Four cases arise: 
(I) Every identity in $\Sigma$ is of the form $v=v$. In this case $\mathscr{V}$ is the variety (1) of all groupoids.

(II) In the notation of Lemma 3 every identity in $\Sigma$ is of the form $v\left(x_{1}, \cdots, x_{n}\right)=w\left(x_{f(1)}, \cdots, x_{f(n)}\right)$, where $f$ is the identity permutation on $\{1, \cdots, n\}$. Moreover, there is an identity $v=w$ in $\Sigma$ such that $v, w$ are distinct. In this case, by (2.1) and (2.2) of Lemma 2, $\mathscr{V}$ is the variety (3) of all semigroups.

(III) There is an identity $v\left(x_{1}, \cdots, x_{n}\right)=w\left(x_{f(1)}, \cdots, x_{f(n)}\right)$ in $\Sigma$, where $f$ is not the identity permutation, but $\Sigma$ does not contain the associative law. In this case, by Lemma $3, \mathscr{V}$ is the variety (2) of commutative groupoids.

(IV) There is an identity $v\left(x_{1}, \cdots, x_{n}\right)=w\left(x_{f(1)}, \cdots, x_{f(1)}\right)$ in $\sum$ such that $f$ is not the identity permutation on $\{1, \cdots, n\}$ and at the same time $\Sigma$ contains the associative law. In this case $\mathscr{V}$ is contained in, and hence by Theorem 2 , equal to the variety (4) of commutative semigroups.

An application.

Let $\mathscr{K}$ be a class of groupoids. By $S(\mathscr{K}), H(\mathscr{K}), \Pi(\mathscr{K})$ we denote respectively the classes of all subgroupoids, all homomorphic images and all cartesian products of groupoids in $\mathscr{K}$. By $P(\mathscr{K})$ we denote the image of $\mathscr{K}$ under $P$ while $[\mathscr{K}]^{0}$ will denote the closure of $\mathscr{K}$ under ()$^{0}$. Let $S_{0}, S_{+}, G_{0}, G_{+}$be the groupoids on the two element set $\{a, b\}$ defined as follows:

$S_{0}$ is the two element semilattice

$S_{+}$is the semigroup satisfying $x y=x^{*}$ identically,

$G_{0}$ is the groupoid with: $a^{2}=b, b^{2}=a, a b=b a=a$

$G_{+}$is the groupoid with: $a^{2}=b, b^{2}=a, a b=a, b a=b$.

As an application of Theorems 1,2 we now have

THEOREM 4. The four varieties (1)-(4) of Theorem 1 are respectively given by: $H S \Pi P \Pi P\left(\left[G_{+}\right]^{0}\right), H S \Pi P$ ПP([G $\left.]_{0}^{0}\right), H S \Pi P \Pi P\left(\left[S_{+}\right]^{0}\right), H S \Pi P \Pi P\left(S_{0}\right)$.

Proof. We need Corollary 2 of Shafaat (to appear) which states that $H S \Pi P \Pi P(\mathscr{K})$ is the $P$-closed variety generated by $\mathscr{K}$. Also, it is easily verified that $H S \Pi\left([\mathscr{K}]^{0}\right)$ is the ()$^{0}$-closed variety generated by $\mathscr{K}$ and hence that $\mathscr{V}=H S \Pi P \Pi P\left(\left[G_{+}\right]^{0}\right)$ is the $P^{0}$-closed variety generated by the groupoid $G_{+}$. By Theorem 1, $\mathscr{V}$ must be one of the four varieties (1)-(4). Since, however, $G_{+}$ is neither associative nor commutative therefore $\mathscr{V}$ must be the variety (1) of all groupoids. The proof for the other varieties is similar.

Finally I should like to thank Professor W. S. Hatcher for bringing Theorem 3 to my notice. 


\section{References}

M. N. Bleicher, H. Schneider and R. L. Wilson (1973), 'Permanence of identities on algebras', Algebra Universalis 3, 72-93.

P. M. Cohn (1965), Universal Algebra (Harper and Row, New York, 1965).

S. Whitney (to appear), Model classes of linear theories.

A. Shafaat (to appear), Power algebras of free algebras.

Département de Mathématiques

Faculté des Sciences

Université Laval

Québec G1K 7P4

Canada. 